\title{
ON MINIMAL SETS OF GENERATORS FOR PRIMITIVE ROOTS
}

\author{
FRANCESCO PAPPALARDI
}

\begin{abstract}
A conjecture of Brown and Zassenhaus (see [2]) states that the first $\log p$ primes generate a primitive root $(\bmod p)$ for almost all primes $p$. As a consequence of a Theorem of Burgess and Elliott (see [3]) it is easy to see that the first $\log ^{2} p \log \log ^{4+\epsilon} p$ primes generate a primitive root $(\bmod p)$ for almost all primes $p$. We improve this showing that the first $\log ^{2} p / \log \log p$ primes generate a primitive root $(\bmod p)$ for almost all primes $p$.
\end{abstract}

For a given odd prime number $p$, we define the function $\kappa$ as

$$
\kappa(p)=\min \left\{r \mid \text { the first } r \text { primes generate } \mathbb{F}_{p}^{*}\right\} .
$$

In $1969, \mathrm{H}$. Brown and $\mathrm{H}$. Zassenhaus conjectured in [2] that $\kappa(p) \leq[\log p]$ with probability almost equal to one.

If we denote by $g(p)$ the least primitive root modulo $p$, then a Theorem of D. A. Burgess and P. D. T. A. Elliott states that

$$
\pi(x)^{-1} \sum_{p \leq x} g(p) \ll \log ^{2} x(\log \log x)^{4} .
$$

If $U$ is the number of primes up to $x$ for which $g(p) \geq T$, then

$$
U T \ll \sum_{p \leq x} g(p) \ll \pi(x) \log ^{2} x(\log \log x)^{4} .
$$

For any $\epsilon>0$, we choose $T=\log ^{2} x(\log \log x)^{4+\epsilon / 2}$ so that $U=o(\pi(x))$ and since $g(p) \leq T$ is product of primes less that $T$, we deduce that for almost all primes $p \leq x$,

$$
\kappa(p) \leq \log ^{2} x(\log \log x)^{4+\epsilon / 2} \leq \log ^{2} p(\log \log p)^{4+\epsilon} .
$$

We will prove the following:

THEOREM 1. Let $\pi$ be the prime counting function. For all but

$$
O\left(\frac{x}{\exp \left\{\frac{(\log \log \log x)^{3} \log x}{4(\log \log x)^{3}}\right\}}\right)
$$

Supported in part by C.N.R.

Received by the editors June 23, 1994; revised October 24, 1994.

AMS subject classification: Primary: 11N56; secondary: 11 A07.

Key words and phrases: sieve theory, primitive roots, Riemann hypothesis.

(c) Canadian Mathematical Society, 1995. 
primes $p \leq x$, we have that

$$
\kappa(p) \leq \pi\left(\frac{\log ^{2} p}{e^{2}} \exp \left\{2 \frac{(\log \log \log p)^{3}}{(\log \log p)^{2}}\right\}\right) .
$$

The proof is based on a uniform estimate for the size of the set

$$
\mathcal{H}_{m, r}(x)=\#\left\{p \leq x|| \Gamma_{r} \mid=\frac{p-1}{m}\right\}
$$

where $m$ and $r$ are given integers strictly greater than one, and

$$
\Gamma_{r}=\left\langle p_{1}, \ldots, p_{r}(\bmod p)\right\rangle
$$

is the subgroup of $\mathbb{F}_{p}^{*}$ generated by the first $r$ primes.

As a subgroup of the cyclic group $\mathbb{F}_{p}^{*}$ with index $m, \Gamma_{r}$ is the subgroup of $m$-th powers $(\bmod p)$. Hence

$$
\mathcal{H}_{m, r}(x)=\left\{p \leq x \mid p \equiv 1(\bmod m) \text { and } p_{i} \text { is an } m \text {-th power }(\bmod p) \forall i=1, \ldots, r\right\} .
$$

If $n_{m}(p)$ is the least prime which is not congruent to an $m$-th power $(\bmod p)$, then we can also write:

$$
\mathcal{H}_{m, r}(x)=\left\{p \leq x \mid p \equiv 1(\bmod m) \text { and } n_{m}(p)>p_{r}\right\} .
$$

We will need to use the large sieve inequality, the proof of which can be found in [1]. That is:

LEMma 2 (The LARGE SIEVE). Let $\mathcal{N}$ be a set of integers contained in the interval $\{1, \ldots, z\}$ and for any prime $p \leq x$, let $\Omega_{p}=\{h(\bmod p) \mid \forall n \in \mathcal{N}, n \not \equiv h(\bmod p)\}$ and

$$
L=\sum_{q \leq x} \mu^{2}(q) \prod_{p \mid q} \frac{\left|\Omega_{p}\right|}{p-\left|\Omega_{p}\right|},
$$

then

$$
|\mathcal{N}| \leq \frac{z+3 x^{2}}{L}
$$

In our case, let $\mathcal{N}=\left\{n \leq z|\forall q| n, q<p_{r}\right\}$ and note that if $p \in \mathcal{H}_{m . r}(x)$, then

$$
\Omega_{p} \supset\{h(\bmod p) \mid h \text { is not an } m \text {-th power }(\bmod p)\}
$$

therefore, for such $p$ 's, $\left|\Omega_{p}\right| \geq p-1-(p-1) / m$ and

$$
L \geq \sum_{p \in \mathcal{H}_{m, r}(x)} \frac{\left|\Omega_{p}\right|}{p-\left|\Omega_{p}\right|} \geq \frac{m-1}{2}\left|\mathcal{H}_{m, r}(x)\right| .
$$

If we let $\Psi(s, t)$ denote the number of integers $n \leq s$ free of prime factors exceeding $t$, then

$$
\mathcal{H}_{m, r}(x) \leq \frac{8 x^{2}}{(m-1) \Psi\left(x^{2}, p_{r}\right)} .
$$

Estimating the function $\Psi(z, y)$ is a classical problem in Number Theory. In 1983, R. Canfield, P. Erdős and C. Pomerance (see [4]) proved the following: 
LEMMA 3. Let $u=\frac{\log z}{\log y}$. There exists an absolute constant $c_{1}$ such that

$$
\Psi(z, y) \geq z \exp \left\{-u\left(\log u+\log \log u-1+\frac{(\log \log u)-1}{\log u}+c_{1} \frac{(\log \log u)^{2}}{\log ^{2} u}\right)\right\}
$$

for all $z \geq 1$ and $u \geq e^{e}$.

Applying Lemma 3 with $z=x^{2}$ and $y=p_{r}$, we get the following:

LEMMA 4. Let $u=2 \log x / \log p_{r}$. There exists an absolute constant $c_{1}$ such that

$$
\mathcal{H}_{m, r}(x) \leq \frac{8}{m} \exp \left\{u\left(\log u+\log \log u-1+\frac{(\log \log u)-1}{\log u}+c_{1} \frac{(\log \log u)^{2}}{\log ^{2} u}\right)\right\},
$$

for all $x \geq 1$ and $u \geq e^{e}$.

Proof OF THEOREM 1. Let us take $p_{r}$ is the range

$$
\log ^{2} x \geq p_{r} \geq \frac{\log ^{2} x}{e^{2}} \exp \left\{\frac{(\log \log \log x)^{3}}{(\log \log x)^{2}}\right\} .
$$

If we set $\log _{2} x=\log \log x, \log _{3} x=\log \log \log x$ and $u=2 \frac{\log x}{\log p_{r}}$, then we can write the estimates:

$$
\begin{gathered}
\frac{\log x}{\log _{2} x} \leq u \leq \frac{\log x}{\log _{2} x-1+\log _{3}^{3} x / 2 \log _{2}^{2} x} \\
\log _{2} x-\log _{3} x \leq \log u \leq \log _{2} x-\log _{3} x+\frac{1}{\log _{2} x} \\
\log _{2} u \leq \log _{3} x-\frac{\log _{3} x}{\log _{2} x}+c_{2} \frac{\log _{3}^{2} x}{\log _{2}^{2} x} \\
\frac{1}{\log _{2} x}-\frac{2}{\log _{2}^{3} x} \leq \frac{1}{\log u} \leq \frac{1}{\log _{2} x}+c_{3} \frac{\log _{3} x}{\log _{2}^{2} x}
\end{gathered}
$$

where $c_{2}$ and $c_{3}$ are absolute constants.

Now let us apply Lemma 4 and deduce that

$$
\begin{aligned}
m \mathcal{H}_{m, r}(x) & \ll \exp \left\{\log x \frac{\log _{2} x-1+c_{4} \frac{\log _{3}^{2} x}{\log _{2}^{2} x}}{\log _{2} x-1+\log _{3}^{3} x / 2 \log _{2}^{2} x}\right\} \\
& \ll \exp \left\{\log x\left(1-\frac{\log _{3}^{3} x}{2 \log _{2}^{3} x}+c_{5}\left(\frac{\log _{3}^{2} x}{\log _{2}^{3} x}\right)\right)\right\}
\end{aligned}
$$

where $c_{4}$ and $c_{5}$ are absolute constants.

Now we are ready to estimate

$$
\#\left\{p \leq x \mid\left[\mathbb{F}_{p}^{*}: \Gamma_{r}\right]>1\right\} .
$$

We note that the index $\left[\mathbb{F}_{p}^{*}: \Gamma_{r}\right]$ is at most $x$ as it is a divisor of $p-1$. 
Since for all but $\mathrm{O}\left(x / \exp \frac{\log x}{\log \log x}\right)$ primes $p$, we may assume that

$$
p>x / \exp (2 \log x / \log \log x)
$$

if we set $p_{r} \geq \frac{\log ^{2} p}{e^{2}} \exp \left(2 \log _{3}^{3} p / \log _{2}^{2} p\right)$ then $p_{r}$ is in the range of (1) and by (2) the number of such primes $p$ for which $\left[\mathbb{F}_{p}^{*}: \Gamma_{r}\right]>1$ is

$\ll \sum_{m=2}^{x} \mathcal{H}_{m, r}(x) \leq\left(\sum_{m=2}^{x} \frac{1}{m}\right) \exp \left\{\log x\left(1-\frac{\log _{3}^{3} x}{2 \log _{2}^{3} x}+c_{5}\left(\frac{\log _{3}^{2} x}{\log _{2}^{3} x}\right)\right)\right\}=\mathrm{O}\left(\frac{x}{\exp \left\{\frac{\log ^{2} \log _{3}^{3} x}{4 \log _{2}^{3} x}\right)}\right)$

and this completes the proof.

ACKNOWLEDGMENTS. A version of Lemma 4 has been proven recently also by S. Konyagin and C. Pomerance in [5].

I would like to thank Professor Ram Murty for his suggestions and for a number of interesting observations.

\section{REFERENCES}

1. E. Bombieri, Le grande crible dans la théorie analytique des nombres, Astérisque 18(1974).

2. H. Brown and H. Zassenhaus, Some empirical observation on primitive roots, J. Number Theory $3(1971)$ 306-309.

3. D. A. Burgess and P. D. T. A. Elliott, The average of the least primitive root, Mathematika 15(1968), 39-50.

4. E. R. Canfield, P. Erdös and C. Pomerance, On a problem of Oppenheim concerning "Factorization Numerorum", J. Number Theory 17(1983), 1-28.

5. S. Konyagin and $\mathrm{C}$. Pomerance, On primes recognizable in deterministic polynomial time, preprint.

\section{Dipartimento di Matematica}

Terza Università degli Studi di Roma

Via Corrado Segre, 4

Roma

00146-Italia

e-mail: pappa@mat.uniroma3.it 